\title{
Accelerated ischemic vascular retinopathy after intravitreally injected bevacizumab for central retinal vein occlusion in elderly patients
}

This article was published in the following Dove Press journal:

Clinical Ophthalmology

28 February 2013

Number of times this article has been viewed

Vincenzo Isola'

Alfredo Pece ${ }^{1,2}$

Claudio Massironi'

Simone Reposi'

Fabio Dimastrogiovanni'

'Department of Ophthalmology, Melegnano Hospital, ${ }^{2}$ Fondazione Retina 3000, Milano, Italy
Correspondence: Dr Vincenzo Isola Retina and Angiography Service, Department of Ophthalmology, Melegnano Hospital, Via Pandina I, 20077, Vizzolo Predabissi, Milan, Italy

Tel +399805 2267

Fax +39 98052267

Email vincenzo.isola@gmail.com
Background: Ischemic changes in the retinal circulation are an uncommon but severe adverse vascular reaction to intravitreal bevacizumab (Avastin ${ }^{\circledR}$, Genentech, San Francisco, CA, USA/ Roche, Basel, Switzerland) for central retinal vein occlusion (CRVO). In the two cases reported here, ischemic changes in the retina vasculature following intravitreal bevacizumab for CRVO were observed with the aim of describing the clinical and angiographic features of these changes.

Methods: Two elderly patients with recent-onset CRVO received one off-label intravitreal injection of bevacizumab $0.05 \mathrm{~mL} / 1.25 \mathrm{mg}$.

Results: In Case 1, the patient's pre-treatment visual acuity was 20/400. At 3 weeks post injection, the patient could count fingers at a distance of $1 \mathrm{ft}(30 \mathrm{~cm})$ and fluorescein angiography showed reduction in intraretinal hemorrhages and areas of retinal non-perfusion. However, at 6 weeks these were markedly increased compared with those seen in the photograph taken 3 weeks after treatment. In Case 2, the patient's pre-treatment visual acuity was 20/200. At 1 month post injection, vision had decreased to 20/400 and fluorescein angiography showed severe macular ischemia with a remarkable capillary dropout throughout the macula.

Conclusion: Ischemic retinal injury may be an uncommon but severe adverse vascular reaction to intravitreal bevacizumab for CRVO. Although progression of retinal ischemia in CRVO could be observed shortly after intravitreal bevacizumab, whether this is a drug- or procedure-related effect or part of the natural history of the condition remains uncertain.

Keywords: Avastin, ischemia, macular infarction, intraretinal hemorrhage, retinal non-perfusion

\section{Introduction}

Early studies indicated that intravitreal injections of bevacizumab (Avastin ${ }^{\circledR}$, Genentech, San Francisco, CA, USA/Roche, Basel, Switzerland) appeared to be safe and effective for the management of central retinal vein occlusion (CRVO) to reverse macular edema. ${ }^{1,2}$ However, clinical and experimental investigations have reported possible circulatory changes as a complication of intravitreal bevacizumab (IVB); these consist of significant depletion of choriocapillaris in primate eyes, progression of capillary non-perfused areas in rabbit eyes, and multiple retinal hemorrhages in eyes with diabetic retinopathy. ${ }^{3,4}$

This report documents two cases of CRVO that rapidly developed retinal ischemic injury with closure of macular capillaries after IVB.

\section{Case I}

A 76-year-old woman was referred to the Retina and Angiography Service of the Department of Ophthalmology, Melegnano Hospital, Milan, Italy, with an 8-week history of reduced vision in her left eye due to CRVO. The patient's medical history 
was remarkable for systemic hypertension, hypertriglyceridemia, and hypercholesterolemia. Visual acuity measured 20/400 in the left eye. Tonometry was $18 \mathrm{mmHg}$ bilaterally. No relative afferent pupillary defect was evident. Slit-lamp biomicroscopy showed no iris or angle neovascularization in either eye. Fundus examination revealed extensive intraretinal hemorrhage and fluorescein angiography (FA) revealed blockage of fluorescence by hemorrhage (Figure 1A and B). Optical coherence tomography (OCT) (Figure 1C) revealed a marked central macular thickening $(689 \mu \mathrm{m})$ with cystoid edema. IVB $(1.25 \mathrm{mg} / 0.05 \mathrm{~mL})$ was administered.

Upon follow-up 3 weeks later, the patient was able to count fingers at a distance of $1 \mathrm{ft}(30 \mathrm{~cm})$. Repeat angiography disclosed capillary non-perfusion in the midperiphery of the retina and in the macular region (Figure 1D), with decreased edema with a central macular thickness of $343 \mu \mathrm{m}$ (Figure 1E).

Six weeks after her initial presentation, the patient's ischemia had become progressively worse, with marked rarefaction of the capillary network, especially at the posterior pole of the retina (Figure 1F). OCT (Figure 1G) demonstrated spontaneous resolution of cystoid edema and macular thickening with no improvement in acuity. Laser photocoagulation was subsequently applied to areas of the retina that were ischemic to prevent development of neovascular glaucoma.

At follow-up 15 months after laser treatment, the patient's visual acuity was counting fingers at $1 \mathrm{ft}$. No iris or angle neovascularization was observed; however, angiography (Figure $1 \mathrm{H}$ ) revealed vascular remodelling with significant capillary dropout around the optic disc and non-perfusion at the posterior pole. Although there was no intraretinal fluid on OCT (Figure 1I), there was significant sub- and intraretinal fibrosis of the fovea and loss of the overlying neurosensory retinal layers.

\section{Case 2}

A 71-year-old man with a 2-month history of CRVO and subjective decreased vision in the right eye was referred to the Retina and Angiography Service for management. The patient had a history of hypertension, obesity, and previous tobacco use. Visual acuity was 20/200 in the right eye and tonometry was $14 \mathrm{mmHg}$. No relative afferent pupillary defect was evident. Anterior segment slit-lamp biomicroscopy did not show iris or angle neovascularization. FA on presentation revealed significant vascular tortuosity and intraretinal hemorrhage without extensive capillary non-perfusion, either within or outside the macula
(Figure 2A and B). As significant cystoid edema $(757 \mu \mathrm{m})$ was evident on OCT (Figure 2C), IVB (1.25 mg/0.05 mL) was injected in the right eye.

At 1 month after the injection, the patient's visual acuity had decreased to 20/400. Repeat FA revealed severe macular ischemia with marked capillary non-perfusion throughout the macula (Figure 2D and E). Follow-up OCT (Figure 2F) showed partial resolution of intraretinal fluid and cysts with reduced macular thickness $(396 \mu \mathrm{m})$. The patient declined further treatment and was lost to follow-up.

However, 14 months later, he was referred back to the retina service, having refused all treatment in the interim. He could count fingers at a distance of $1 \mathrm{ft}$ without improvement with pinhole. Angiography showed leakage with dye pooling in the central macula (Figure $2 \mathrm{G}$ ) and some increase in the extent of capillary non-perfusion (Figure $2 \mathrm{H}$ ) temporal and inferior to the macular region. OCT (Figure 2I) revealed chronic, persistent macular edema and thickening.

\section{Discussion}

The reported cases show the clinical and angiographic findings of CRVO eyes, which are suggestive of accelerated ischemic vascular retinopathy after anti-vascular endothelial growth factor (VEGF) treatment. It may be speculated that the development of ischemic changes is unrelated to bevacizumab and is instead part of the natural course of the disease. In fact, the cumulative chances of conversion of non-ischemic CRVO to ischemic CRVO during follow-up have been estimated by Hayreh et al in a large series of eyes with nonischemic CRVO. ${ }^{5}$ From the time of onset of non-ischemic CRVO, conversion occurred within 6 months in $9.4 \%$ of eyes and reached near its maximum within 18 months in $12.6 \%$ of eyes. In our cases, the retinal capillary bed filled well when the eyes were first seen at baseline. However, when seen 3 weeks (Case 1) and 1 month (Case 2) later, the retinal capillary beds were completely obliterated angiographically. Fluorescein angiograms did confirm that the progression of the ischemia was very rapid and occurred soon after bevacizumab administration. Fortunately, the combination of ischemic CRVO with anti-VEGF therapy is an uncommon occurrence that might be a drug-related adverse event, as recently reported by Kim et al in their article describing a case of non-ischemic CRVO conversion to ischemic CRVO 3 months after IVB. ${ }^{6}$

Shimura et al described two cases of progression of macular ischemia despite an improvement in macular edema after IVB for CRVO in patients with poor glycemic control of their diabetes and a history of systemic vascular disorder. ${ }^{7}$ 

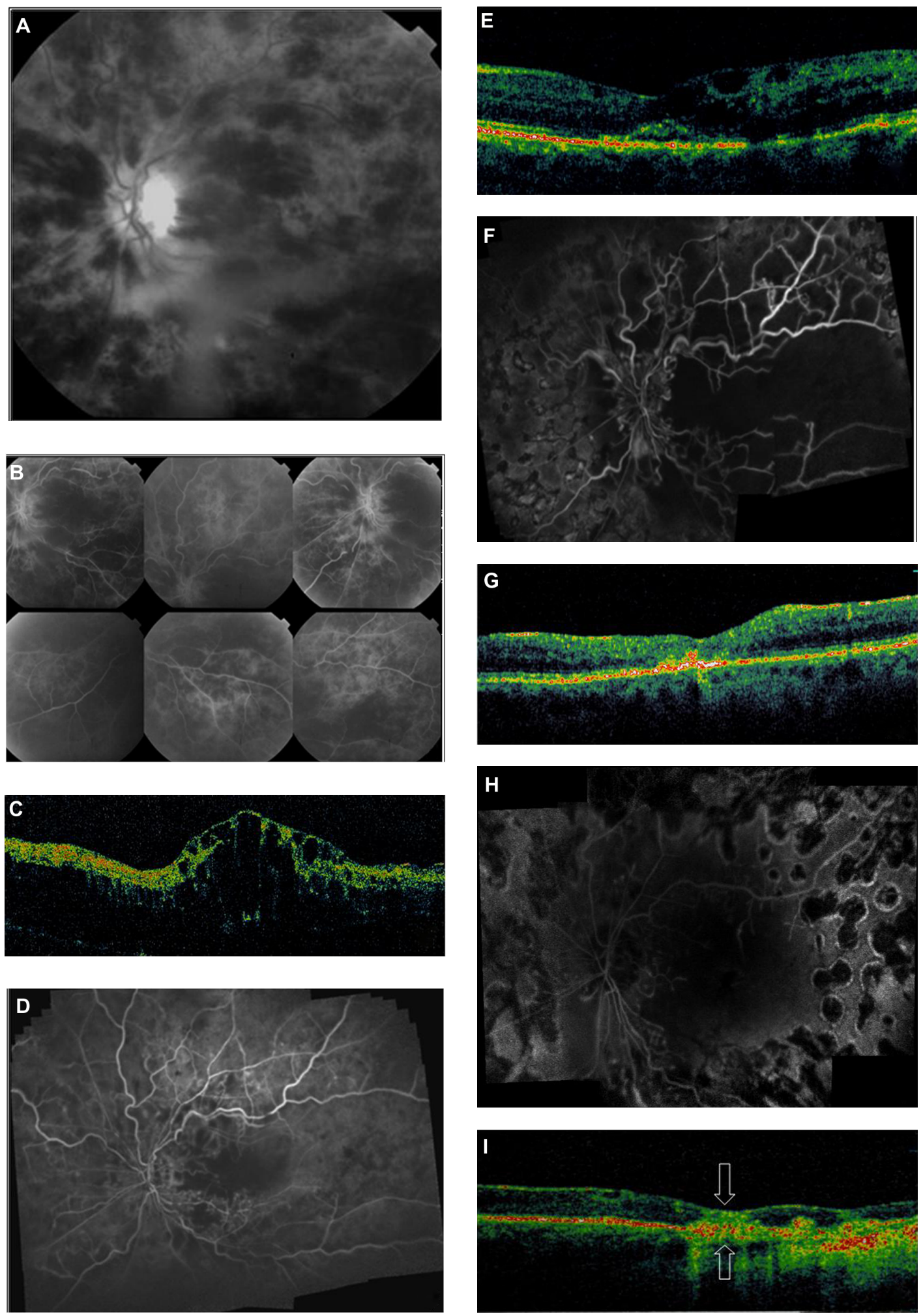

Figure I Case I. Baseline black and white fundus photograph (A) and fluorescent angiography (FA) image (B) showing central retinal vein occlusion with diffuse intraretinal hemorrhages and edema prior to intravitreal bevacizumab injection. (C) Optical coherence tomography (OCT) image showing central macular thickening with cystoid edema. (D) FA image 3 weeks after bevacizumab injection showing a reduction of intraretinal blood and areas of retinal non-perfusion. (E) Follow-up OCT image shows reduced macular thickness with some cysts and intraretinal fluid. (F) FA image 6 weeks after injection showing areas of capillary non-perfusion and retinal ischemia markedly increased compared with at 3 weeks (see D). (G) OCT image showing spontaneous resolution of cystoid edema and macular thickening with no improvement in visual acuity. (H) Angiography image at 15 months follow-up. Pan-retinal laser photocoagulation has been performed. There is significant capillary non-perfusion at the posterior pole. (I) OCT image indicates the absence of intraretinal fluid, with multifocal points of hyperreflectivity above the retinal pigment epithelium/choriocapillaris layer. Mild sub- and intraretinal fibrosis can be observed in the fovea (arrows) as a thickened, highly reflective, yellow-green, and reddish band with loss of the overlying neurosensory retinal layers. 

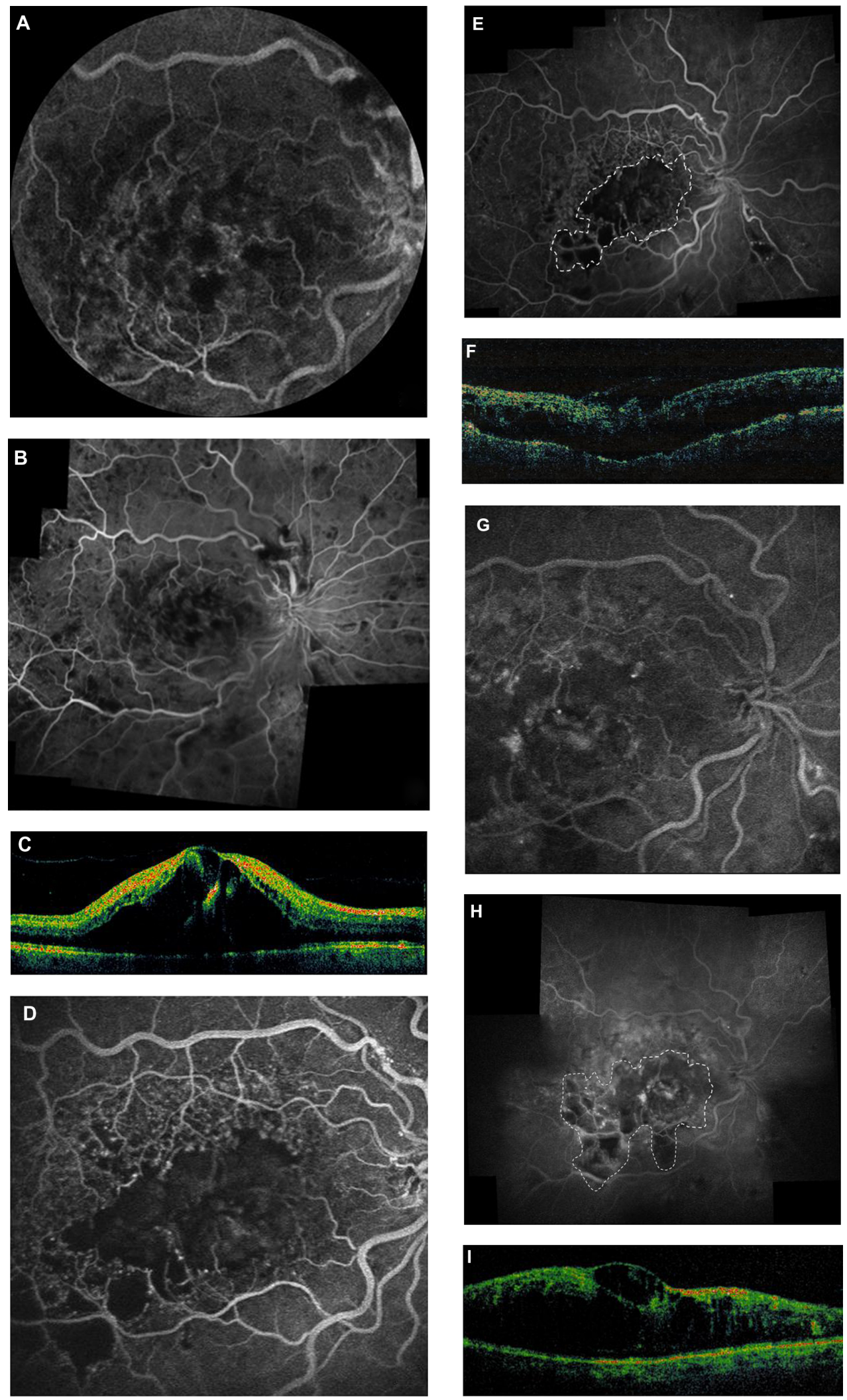

Figure 2 Case 2. Baseline fluorescein angiography images (A and B) of central retinal vein occlusion showing patchy areas of hypofluorescence corresponding to intraretinal hemorrhages and dye leakage from increased capillary permeability in the macula. (C) Optical coherence tomography (OCT) image showing significant cystoid edema. (D and E) Angiography images 4 weeks after injection of bevacizumab. Images show severe macular ischemia, with marked capillary dropout throughout the macula (dashed line). Acuity has dropped to 20/400. (F) OCT image at 4 weeks showing partial resolution of intraretinal fluid and cysts with reduced macular thickness. (G) Persistent fluorescein leakage and pooling of dye in the central macula 14 months post injection. $(\mathbf{H})$ Angiography image at I4 months post injection showing interval increase in the extent of capillary non-perfusion temporal and inferior to the macula (dashed line). (I) OCT image showing persistent macular thickening and large cystic changes. 
Furino et al reported one case of severe visual loss due to hemorrhagic macular infarction 3 weeks after bevacizumab was injected in the CRVO-affected eye. ${ }^{8}$ Nowadays, however, the current literature does not provide information about the incidence rate of retinal ischemia after IVB for CRVO.

This vascular change has been found at our institution in $3.0 \%$ (2/66 eyes) of patients treated for macular edema as a result of CRVO (September 2006 to May 2009). This indicates that such an event could also interfere with the continuation of intravitreal therapy with bevacizumab, especially in eyes with clinical and angiographic features of hypoperfusion in the retinal circulation. At present, the role of bevacizumab in the development of capillary dropout is still poorly understood and the mechanism of action is not clear.

Boyd et al suggested that immediately after the onset of ischemic CRVO, patients had an initial transient elevation of VEGF concentration that may reflect the retinal cells' immediate response to the drop in oxygen saturation. ${ }^{9}$ In such cases, VEGF may have an important function in capillary remodelling for revascularizing the areas of ischemia with insufficient retinal oxygenation. Consequently, VEGF suppression with monoclonal antibodies could be dangerous in the acute phase of CRVO because of blockade of early neuroprotective effect. It could also delay the development of VEGF-mediated retinal venogenesis as a short term for retino-ciliary collateral formations from pre-existing venous channels for the creation of compensatory blood vessels that can effectively bypass the venous occlusion's point.

Similarly, the expression of VEGF is essential for the growth of coronary collateral vessels after myocardial infarction and for the reduction of infarct size in the ischemic brain. ${ }^{10,11}$ Another possible explanation is that the ischemic circulatory changes could be a complication resulting from elevated, transient intraocular pressure spikes. ${ }^{12}$

We recommend careful clinical and angiographic observation to differentiate between ischemic and non-ischemic CRVO before considering the therapeutic intravitreal inhibition of VEGF activity. The first crucial step in the management of CRVO is to find out what type of CRVO an eye has because the prognosis, management, and outcome of the two forms are completely different. A non-ischemic CRVO is a comparatively benign disease - with visual reduction from cystoid macular edema the major complication - that can be treated with anti-VEGF therapy. This type of CRVO does not develop the most serious complication of intraocular neovascularization. In contrast, ischemic CRVO in eyes with 75 disc diameters or more (ie, eyes that show virtually no intact capillaries in the posterior pole) of retinal ischemia are at highest risk of blinding diseases and anterior segment neovascularization leading to neovascular glaucoma. ${ }^{13}$ The Central Retinal Vein Occlusion Study Group guidelines recommend that wide areas of retinal capillary non-perfusion be treated with trans-pupillary thermal laser pan-retinal photocoagulation to prevent the development of neovascular glaucoma. ${ }^{14}$ Although progression of retinal ischemia in CRVO could be observed shortly following IVB in both cases discussed in this article, whether this is a drugor procedure-related effect or part of the natural history of the condition remains uncertain.

Further study is necessary to determine whether the benefit of bevacizumab in eyes with fairly severe non-ischemic CRVO outweighs the possible risks such as the one reported in this paper.

\section{Disclosure}

This study was supported by Fondazione Retina 3000, Milan, Italy. The authors report no other conflicts of interest in this work.

\section{References}

1. Iturralde D, Spaide RF, Meyerle CB, et al. Intravitreal bevacizumab (Avastin) treatment of macular edema in central retinal vein occlusion: a short-term study. Retina. 2006;26(3):279-284.

2. Kriechbaum K, Michels S, Prager F, et al. Intravitreal Avastin for macular oedema secondary to retinal vein occlusion: a prospective study. Br J Ophthalmol. 2008;92(4):518-522.

3. Peters S, Heiduschka P, Julien S, et al. Ultrastructural findings in the primate eye after intravitreal injection of bevacizumab. Am J Ophthalmol. 2007;143(6):995-1002.

4. Lee CS, Koh HJ. Multiple retinal haemorrhages in diabetic retinopathy after adjunctive intravitreal bevacizumab (Avastin) with pars plana vitrectomy. Acta Ophthalmol. 2008;86(7):812-813.

5. Hayreh SS, Zimmerman MB, Podhajsky P. Incidence of various types of retinal vein occlusion and their recurrence and demographic characteristics. Am J Ophthalmol. 1994;117(4):429-441.

6. Kim KS, Chang HR, Song S. Ischaemic change after intravitreal bevacizumab (Avastin) injection for macular oedema secondary to nonischaemic central retinal vein occlusion. Acta Ophthalmol. 2008;86(8): 925-927.

7. Shimura M, Yasuda K. Macular ischaemia after intravitreal bevacizumab injection in patients with central retinal vein occlusion and a history of diabetes and vascular disease. Br J Ophthalmol. 2010;94(3): 381-383.

8. Furino C, Boscia F, Cardascia N et al. Hemorrhagic macular infarction after intravitreal bevacizumab for central retinal vein occlusion. Ophthalmic Surg Lasers Imaging. 2010. Epub Mar 9.

9. Boyd SR, Zachary I, Chakravarthy U, et al. Correlation of increased vascular endothelial growth factor with neovascularization and permeability in ischemic central vein occlusion. Arch Ophthalmol. 2002; 120(12):1644-1650.

10. Matsunaga T, Warltier DC, Tessmer J et al. Expression of VEGF and angiopoietins-1 and -2 during ischemia-induced coronary angiogenesis. Am J Physiol Heart Circ Physiol. 2003;285(1):H352-H358.

11. Sun Y, Jin K, Xie L, et al. VEGF-induced neuroprotection, neurogenesis, and angiogenesis after focal cerebral ischemia. J Clin Invest. 2003; 111(12):1843-1851. 
12. Frenkel MP, Haji SA, Frenkel RE. Effect of prophylactic intraocular pressure-lowering medication on intraocular pressure spikes after intravitreal injections. Arch Ophthalmol. 2010;128(12):1523-1527.

13. The Central Vein Occlusion Study Group N report. A randomized clinical trial of early panretinal photocoagulation for ischemic central vein occlusion. Ophthalmology. 1995;102(10):1434-1444.
14. The Central Vein Occlusion Study Group. Natural history and clinical management of central retinal vein occlusion. Arch Ophthalmol. 1997;115(4):486-491.

\section{Publish your work in this journal}

Clinical Ophthalmology is an international, peer-reviewed journal covering all subspecialties within ophthalmology. Key topics include: Optometry; Visual science; Pharmacology and drug therapy in eye diseases; Basic Sciences; Primary and Secondary eye care; Patient Safety and Quality of Care Improvements. This journal is indexed on
PubMed Central and CAS, and is the official journal of The Society of Clinical Ophthalmology (SCO). The manuscript management system is completely online and includes a very quick and fair peer-review system, which is all easy to use. Visit http://www.dovepress.com/ testimonials.php to read real quotes from published authors. 